\title{
COMPOSICIÓN QUÍMICA Y ENSAYOS DE ACTIVIDAD ANTIOXIDANTE Y DEL EFECTO FUNGISTÁTICO SOBRE Candida albicans DEL ACEITE ESENCIAL DE Piper aduncum L. "MATICO"
}

\author{
Sharon Ingaroca ${ }^{* a}$, Américo Castro $^{\mathrm{a}}$, Norma Ramos ${ }^{\mathrm{a}}$
}

\begin{abstract}
RESUMEN
El aceite esencial se obtuvo de las hojas de Piper aduncum L. procedentes del Departamento de Loreto, mediante destilación por arrastre de vapor de agua con un rendimiento de $1,1 \% \mathrm{v} / \mathrm{p}$. La composición química se determinó mediante Cromatografía de Gases / Espectrometría de Masas (CG/EM), identificándose 35 componentes químicos, de los cuales ocho representan el $76,53 \%$ del área total. Los principales compuestos fueron fenilpropanoides $(1,2$, 4-trimetoxi-5-(1-propenil)- benceno (39,32\%) y metileugenol $(12,85 \%)$ ), hidrocarburos sesquiterpenoides (germacreno D (7,52\%), biciclogermacreno $(5,88 \%), \beta$-cariofileno $(2,82$ $\%)$ y $\delta$ - cadineno $(2,81 \%))$, un hidrocarburo monoterpenoide $(\beta$-ocimeno $(2,34 \%)$ ) y un monoterpenoide oxigenado (4,7,7-trimetilbiciclo [2.2.1] heptan-3-ona (2,99\%)). La actividad antioxidante fue evaluada mediante los métodos de captación de los radicales $\mathrm{DPPH}^{\bullet} \mathrm{y}$ ABTS $^{\bullet+}$. La capacidad antioxidante equivalente a Trolox (TEAC- DPPH) (TEAC-ABTS) fue de 1,839 y 17,79 $\mu \mathrm{g}$ Trolox/mg del aceite esencial, respectivamente. El efecto fungistático fue evaluado mediante el método de microdilución colorimétrica en caldo, mostrando actividad con un MIC de 1,24 mg/mL. Los resultados demuestran que a pesar de que la actividad antioxidante y el efecto fungistático del aceite esencial fueron limitados; el alto contenido de fenilpropanoides lo hace un candidato prometedor para futuras investigaciones.
\end{abstract}

Palabras clave: Piper aduncum L., antioxidante, fungistático, Candida albicans.

a Instituto de Investigación en Ciencias Farmacéuticas y Recursos Naturales “Juan de Dios Guevara”, Facultad de Farmacia y Bioquímica, Universidad Nacional Mayor de San Marcos, Lima-Perú. sharoningarocatejedafyb@, gmail.com 


\title{
CHEMICAL COMPOSITION, ANTIOXIDANT ACTIVITY AND FUNGISTATIC EFFECT AGAINST Candida albicans OF THE ESSENTIAL OIL OF Piper aduncum L. "MATICO"
}

\begin{abstract}
The essential oil was obtained from leaves of Piper aduncum L. "matico" from in Department of Loreto by steam distillation with a yield of $1.1 \% \mathrm{v} / \mathrm{w}$. The chemical composition has determined by chromatography/mass spectrometry (GC/MS). Thirty-five chemical components were identified, of which eight represent $76,53 \%$ of the total area. The main volatile compounds are phenylpropanoids (1, 2, 4-trimethoxy-5- (1-propenyl) -benzene $(39,32 \%)$ and methyleugenol $(12,85 \%))$, sesquiterpenoid hydrocarbons (germacrene D $(7,52 \%)$, bicyclogermacrene $(5,88 \%), \beta$-caryophyllene $(2,82 \%)$ and $\delta$-cadinene $(2,81 \%))$, a monoterpenoid hydrocarbon $(\beta$-ocimene $(2,34 \%))$ and an oxygenated monoterpenoid (4,7,7-trimethylbicyclo [2.2.1] heptan-3-one (2,99\%)). The antioxidant activity was evaluated by $\mathrm{DPPH}^{\bullet}$ and $\mathrm{ABTS}^{\bullet+}$ radical scavenging methods. The antioxidant capacity equivalent to Trolox (TEAC-DPPH) (TEAC-ABTS) was 1.839 and $17.79 \mu \mathrm{g}$ Trolox/mg of the essential oil, respectively. The fungistatic effect was evaluated by colorimetric broth microdilution method, showing activity with a MIC of $1.24 \mathrm{mg} / \mathrm{mL}$. The results show that although the antioxidant activity and the fungistatic effect of the essential oil of Piper aduncum L. were limited, however, the high content of phenylpropanoids makes it a promising candidate for future research.
\end{abstract}

Key words: Piper aduncum L., antioxidant, fungistatic, Candida albicans.

\section{INTRODUCCIÓN}

En la actualidad, existe una constante preocupación por la seguridad de los antioxidantes sintéticos utilizados para mantener la calidad de los productos alimenticios, lo que ha motivado a la industria alimentaria a buscar alternativas naturales ${ }^{1}$.

Por otro lado, la candidiasis en la actualidad representa la tercera a cuarta infección nosocomial más frecuente en hospitales en todo el mundo, lo que la ha convertido en un problema de salud pública $^{2}$. Simultáneamente, la resistencia a fármacos antifúngicos también ha aumentado considerablemente en los últimos años ${ }^{3}$. Por lo tanto, se necesitan nuevos compuestos con propiedades antifúngicas para hacer frente al desafió de las micosis invasivas.

Las plantas medicinales constituyen un reservorio de compuestos químicos estructuralmente diversos (compuestos bioactivos), por lo que pueden ser una fuente para el desarrollo de nuevos agentes antimicrobianos y antifúngicos. Los aceites esenciales, son objeto de interés científico en las últimas décadas, con un análisis exhaustivo que indica que muchos de estos y sus componentes aislados poseen diversas actividades ${ }^{4}$. 
En este contexto, la Amazonía peruana, como región fitogeográfica y biodiversa, es una fuente de especies de plantas. Así, la familia Piperaceae, es reconocida en el Perú, por presentar tres géneros y 830 especies, de los cuales el más abundante es el género Piper 5 . Piper aduncum L se usa en la medicina tradicional como antiséptico, antidiarreico, antirreumático, astringente y tónico ${ }^{6}$. Los yaneshas, comunidad nativa de la Amazonía peruana, preparan tés y baños de vapor de las hojas contra infecciones generales y fiebre ${ }^{7}$. Sin embargo, no hay suficientes datos relacionados a sus actividades terapéuticas que validen sus usos tradicionales. En este sentido, el propósito del estudio fue evaluar la composición química, la actividad antioxidante y el efecto fungistático del aceite esencial de Piper aduncum L "matico" frente a Candida albicans.

\section{PARTE EXPERIMENTAL}

\section{Material vegetal}

Las hojas de Piper aduncum L. "matico" fueron colectadas en el Departamento de Loreto, provincia de Maynas, distrito de Napo. Las hojas fueron separadas de los tallos y almacenadas a temperatura ambiente, bajo luz difusa. El peso promedio del material fresco de la planta fue de $5 \mathrm{~kg}$. La clasificación taxonómica se realizó en el Museo de Historia Natural de la Universidad Nacional Mayor de San Marcos.

\section{Obtención del aceite esencial}

El aceite esencial se extrajo en un sistema de destilación por arrastre de vapor de agua hasta su recuperación total. El volumen de aceite esencial obtenido fue deshidratado con sulfato de sodio anhidro, luego fue transferido a un vial ámbar y posteriormente almacenado bajo refrigeración $\left(4^{\circ} \mathrm{C}\right)$ y protegido de la luz, hasta su uso.

\section{Productos químicos}

Todos los componentes usados en este trabajo fueron de grado analítico. Los reactivos y estándares (DPPH, ABTS y Trolox $\left.{ }^{\circledR}\right)$ para la evaluación de la actividad antioxidante fueron comprados de Sigma-Aldrich Chemical Co. (St. Louis, MO, USA). El estándar ketoconazol para la evaluación del efecto fungistático fue suministrado por Laboratorios Induquímica. Los medios de cultivo RPMI 1640 con glutamina sin bicarbonato y agar dextrosa Sabouraud al $4 \%$ fueron adquiridos también por Sigma-Aldrich Chemical Co. y EMD Millipore Co. (Billerica, MA 01821, USA). Los solventes metanol y dimetilsulfoxido (DMSO) fueron también obtenidos de EMD Millipore Co. (Billerica, MA 01821, USA). Otros productos químicos como etanol $96^{\circ}$ y sulfato de sodio anhidro, así como polisorbato 20 (Tween 20) y resazurina fueron proporcionados por el Instituto de Investigación en Ciencias Farmacéuticas y Recursos Naturales "Juan de Dios Guevara" y por el Instituto de Química Biológica, Microbiología y Biotecnología "Marco Antonio Garrido Malo", respectivamente. Agua bidestilada fue usada a lo largo de este trabajo. 


\section{Microorganismo y condiciones de crecimiento}

Candida albicans ATCC 10231 es un cultivo estándar de la Colección Americana de Cultivos Tipo (ATCC) y fue suministrado por el Instituto Investigaciones Estomatológicas de la Facultad de Odontología de la UNMSM. La cepa fue cultivada de stock congelados y conservados en placas con agar dextrosa Sabouraud al $4 \%$ en condiciones aeróbicas a $37^{\circ}$ C durante 48 horas.

\section{Cromatografía de gases / espectrometría de masas (CG/EM)}

El análisis del aceite esencial fue realizado mediante cromatografía de gases acoplado a espectrometría de masas (CG/EM) usando un Agilent Technologies 7890 y un Agilent Technologies 5975C, respectivamente, equipado con una columna capilar de silica gel J\&W 122-1545.67659 DB- $5 \mathrm{~ms}, 325^{\circ} \mathrm{C}: 60 \mathrm{~m}$ x $250 \mu \mathrm{m}$ x 0,25 $\mu \mathrm{m}$. Las condiciones operativas fueron las siguientes: la programación de la temperatura de la columna para CG empezó en $80^{\circ} \mathrm{C}$, luego se incrementó a $10^{\circ} \mathrm{C} /$ min hasta $180^{\circ} \mathrm{C}$, seguida de $2,5^{\circ} \mathrm{C} /$ min hasta $200^{\circ} \mathrm{C}$ por $5 \mathrm{~min}, 2,5^{\circ} \mathrm{C} / \mathrm{min}$ hasta $240^{\circ} \mathrm{C}$ y finalmente $5^{\circ} \mathrm{C} / \mathrm{min}$ hasta $300^{\circ} \mathrm{C}$ manteniéndose por 2 minutos. El gas portador fue helio con una tasa de flujo de $1.0 \mathrm{~mL} / \mathrm{min}$; la temperatura de inyección fue de $300{ }^{\circ} \mathrm{C}$; el volumen de inyección de la muestra fue de $1 \mu \mathrm{L}(20 \mu \mathrm{L}$ de aceite en $1 \mathrm{~mL}$ de diclorometano) y la proporción de división fue de 20:1. El tiempo de corrida fue de 54 minutos. Las condiciones de espectrometría de masas fueron las siguientes: la energía de ionización fue de $70 \mathrm{eV}$ y la temperatura de detección fue de $250{ }^{\circ} \mathrm{C}$.

Los componentes fueron identificados por análisis comparativo de los espectros de masa frente a la base de datos del sistema GC/MS (Nist 08.lib y Flavor 02) junto con los índices de retención (RI) relativos, obtenidos de la inyección de una mezcla de n-alcanos usando la ecuación de Van Den Dool y Kratz.

El contenido relativo de todos los componentes identificados fue estimado por comparación de su área con las áreas totales.

\section{Actividad antioxidante}

El aceite esencial fue analizado mediante dos ensayos. La actividad de captación del radical DPPH se realizó siguiendo el método de Brand- Williams et al. ${ }^{8}$ y la actividad de captación del radical ABTS se realizó según el método de Re et al ${ }^{9}$. La muestra fue diluida con etanol de $96^{\circ}$. El estándar de referencia fue Trolox ${ }^{\circledR}$. Ambos ensayos fueron realizados por triplicado y en un espectrofotómetro UV-VIS (Thermo Scientific TM GENESYS 10S), a $25^{\circ} \mathrm{C}$. Los resultados se expresaron como concentración inhibitoria media (IC50) y como unidades de capacidad antioxidante equivalente a Trolox (TEAC).

\section{Efecto fungistático}

El efecto fungistático del aceite esencial fue evaluado mediante el método de microdilución en placas de 96 pocillos, de acuerdo protocolo M27-A3 ${ }^{10}$ del Instituto de Estándares de Laboratorio Clínico (CLSI) y las modificaciones de Liu M. et al. ${ }^{11}$. La lectura de los resultados se hizo visualmente y se expresaron como concentración mínima inhibitoria (MIC), que se define como la concentración más baja de la muestra a la que no se produce el cambio de color $^{11}$. El promedio de tres valores se reportó como MIC. 


\section{Análisis estadístico}

Los datos recolectados de la actividad antioxidante fueron analizados por análisis de varianza de un factor (ANOVA) usando el software SPSS 23.0 (SPSS Inc., Chicago, IL). La medición de la diferencia se determinó de acuerdo con la prueba de rango múltiple de Tuckey. Los valores de $\mathrm{P}<0,05$ se consideraron estadísticamente significativos. Los ensayos se realizaron por triplicado y los resultados se expresaron como media \pm desviación estándar (DE). Los valores de CI50 se calcularon usando análisis regresión lineal.

\section{RESULTADOS Y DISCUSIÓN}

\section{Composición química y cuantificación del aceite esencial}

El rendimiento (v/p de hojas) fue 1,1 \% v/p. La composición química del aceite esencial de P. aduncum L. se muestra en la tabla 1. Los datos de GC-MS revelaron 35 componentes, de los cuales ocho representan el 76,53 \% del área total del pico en el cromatograma. Los principales componentes fueron 1,2,4-trimetoxi-5-(1-propenil)-benceno (39,32\%), metileugenol (12,85\%), germacreno D (7,52\%), biciclogermacreno (5,88\%), 4,7,7-trimetilbiciclo [2.2.1] heptan-3-ona $(2,99 \%)$ y $\beta$-cariofileno $(2,82 \%), \delta$-cadineno $(2,81 \%)$ y $\beta$-ocimeno $(2,34 \%)$.

Para esta especie se han informado diferentes perfiles químicos. El principal componente en Bolivia fue el monoterpenoide oxigenado 1,8-cineol (40,5\%). Panamá fue abundante en sesquiterpenoides como $\beta$-cariofileno (17.4\%) y aromadendreno (13,4\%). En Cuba, fueron monoterpenoides oxigenados como 4,7,7-trimetilbiciclo [2.2.1] heptan-3-ona (17,1-18,1\%), viridiflorol (14,5\%) y piperitona (23,7\%). Un quimiotipo encontrado con frecuencia en Brasil, Ecuador oriental, sur de Asia oriental y Oceanía fue dillapiol (30-90\%) ${ }^{12}$. Los resultados de este estudio no solo confirman la amplia variabilidad en la composición química de Piper aduncum L. sino también evidencian de manera preliminar un perfil diferente. La existencia de estas variaciones puede atribuirse a las complejas interacciones entre los factores genéticos (variedades, subespecies y quimiotipo), las condiciones ambientales, tiempo de recolección y los métodos de extracción. Así, por ejemplo, el alto contenido de fenilpropanoides, podría estar asociado con una mayor expresión de los genes de la enzima chalcona sintasa en comparación con la enzima terpeno sintasa, productora de monoterpenoides y sesquiterpenoides ${ }^{13}$. 
Tabla 1. Composición química del aceite esencial de Piper aduncum L. realizado por $\mathrm{CG} / \mathrm{EM}$.

\begin{tabular}{|c|c|c|c|}
\hline Número & Componente ${ }^{\mathrm{a}}$ & $\begin{array}{c}\mathbf{t}_{\mathbf{R}}^{\mathbf{b}} \\
(\mathrm{min}) \\
\end{array}$ & $\begin{array}{c}\% \text { en la } \\
\text { muestra }^{c}\end{array}$ \\
\hline 1 & $\beta$-mirceno & 7,23 & 0,32 \\
\hline 2 & $\beta$-pineno & 7,32 & 1,30 \\
\hline 3 & 3-careno & 7,64 & 0,19 \\
\hline 4 & $\beta$-trans-ocimeno & 7,80 & 0,92 \\
\hline 5 & D-limoneno & 7,89 & 0,42 \\
\hline 6 & $\beta$-ocimeno & 7,96 & 2,34 \\
\hline 7 & Terpinoleno & 8,64 & 1,06 \\
\hline 8 & Linalol & 8,70 & 0,53 \\
\hline 9 & 4,7,7-trimetilbiciclo [2.2.1] heptan-3-ona (alcanfor) & 9,68 & 2,99 \\
\hline 10 & Isoborneol & 9,91 & 0,83 \\
\hline 11 & -cimen-8-ol & 10,06 & 0,31 \\
\hline 12 & Elixeno & 12,32 & 0,54 \\
\hline 13 & $\alpha$-cubebeno & 12,54 & 0,28 \\
\hline 14 & $\alpha$-copaeno & 13,11 & 1,49 \\
\hline 15 & Metileugenol & 13,20 & 12,85 \\
\hline 16 & 8-Isopropenil-1,5-dimetil-ciclodeca-1,5-dieno & 13,26 & 0,87 \\
\hline 17 & Desconocido $\left(\mathrm{C}_{15} \mathrm{H}_{24}\right)$ & 13,32 & 0,72 \\
\hline 18 & $\beta$-cariofileno & 14,00 & 2,82 \\
\hline 19 & $\beta$-cubebeno & 14,12 & 0,41 \\
\hline 20 & Aromadendreno & 14,33 & 0,77 \\
\hline 21 & $\alpha$-cariofileno & 14,66 & 1,55 \\
\hline 22 & L-alloaromadendreno & 14,75 & 0,56 \\
\hline 23 & Pentadecano & 14,91 & 3,62 \\
\hline 24 & Germacreno D & 15,12 & 7,52 \\
\hline 25 & $\alpha$-muroleno & 15,30 & 1,11 \\
\hline 26 & Biciclogermacreno & 15,40 & 5,88 \\
\hline 27 & $\delta$-cadineno & 15,67 & 2,81 \\
\hline 28 & Desconocido $\left(\mathrm{C}_{15} \mathrm{H}_{26} \mathrm{O}\right)$ & 15,75 & 1,57 \\
\hline 29 & Elemicina & 15,88 & 0,78 \\
\hline 30 & 1,2,4-trimetoxi-5-(1-propenil)-benceno (asarona) & 16,36 & 39,32 \\
\hline 31 & Germacreno D-4-ol & 17,12 & 0,56 \\
\hline 32 & Espatulenol & 17,18 & 1,41 \\
\hline 33 & Óxido de cariofilleno & 17,37 & 0,56 \\
\hline 34 & $\alpha$-cadinol & 18,90 & 0,31 \\
\hline \multirow[t]{2}{*}{35} & Apiol & 19,04 & 0,48 \\
\hline & Total & & 100 \\
\hline
\end{tabular}

Componentes listados en orden de elución de una columna J\&W 122-1545.67659 DB-5ms.

${ }^{b} t_{R}$, Índices de retención (min) calculados en la columna J\&W 122-1545.67659 DB-5ms relativa a C8-C28 n-alcanos.

${ }^{\text {c }}$ Porcentaje del área relativa (área de pico relativa al área del pico total en el cromatograma). 
Estos componentes pertenecen a seis familias químicas: fenilpropanoides, hidrocarburos sesquiterpenoides, sesquiterpenoides oxigenados, hidrocarburos monoterpenoides, monoterpenoides oxigenados y compuestos alifáticos. Los fenilpropanoides fueron los más abundantes $(53,43 \%)$ con 1,2,4-trimetoxi-5-(1-propenil)-benceno y metileugenol como los componentes principales de este grupo de compuestos químicos. Los terpenoides $(40,66 \%)$ representan el segundo grupo. El contenido de hidrocarburos sesquiterpenoides $(26,61 \%)$ fue más alto que los sesquiterpenoides oxigenados $(2,84 \%)$, mientras que la cantidad de hidrocarburos monoterpenoides $(6,55 \%)$ fue similar al de monoterpenoides oxigenados (4,66\%) (figura 1).

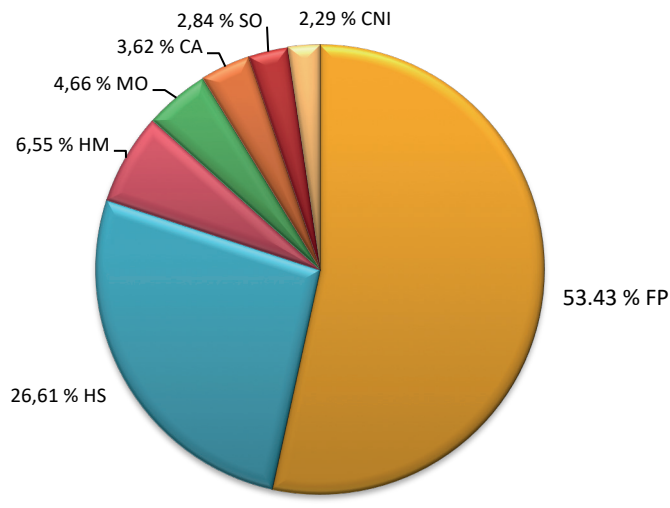

Figura 1. Distribución de los diferentes grupos químicos de los componentes del aceite esencial de $P$. aduncum L. FP: fenilpropanoides; MO: monoterpenoides oxigenados; HM: hidrocarburos monoterpenoides; $\mathrm{SO}$ : sesquiterpenoides oxigenados; HS: hidrocarburos sesquiterpenoides; CA: compuestos alifáticos; CNI: compuestos no identificados.

\section{Actividad antioxidante del aceite esencial}

Los resultados se expresaron mediante valores de $\mathrm{IC}_{50} \mathrm{y}$ en unidades TEAC en las tablas $2 \mathrm{y}$ 3 , respectivamente. La actividad antioxidante por ambos métodos fue baja en comparación con el estándar polifenólico Trolox ${ }^{\circledR}$, análogo soluble de la vitamina E. Esta diferencia puede ser el resultado de diferentes mecanismos relacionados con sus diversas estructuras. En el caso de los polifenoles, está claramente relacionada con los grupos hidroxilos, unidos a un anillo de benceno, mientras que, para los isoprenoides, está relacionada con la presencia de dobles enlaces conjugados ${ }^{14}$. Por lo tanto, esta baja actividad del aceite esencial de Piper aduncum L se atribuiría a la presencia de 4,7,7-trimetilbiciclo [2.2.1] heptan-3-ona, $\beta$-cariofileno, biciclogermacreno, germacreno $\mathrm{D}, \mathrm{y} \beta$-ocimeno, así como a los efectos sinérgicos y antagónicos entre ellos ${ }^{14,15}$. 
Tabla 2. Actividad antioxidante del aceite esencial de Piper aduncum L. expresada como concentración inhibitoria media $\left(\mathrm{IC}_{50}\right)$.

\begin{tabular}{|c|c|c|}
\hline \multirow{2}{*}{ Muestra } & \multicolumn{2}{|c|}{$\mathrm{IC}_{50}(\mu \mathrm{g} / \mathrm{mL})$} \\
\hline & DPPH & $\mathrm{ABTS}^{\bullet+}$ \\
\hline $\begin{array}{l}\text { Aceite esencial de } \\
\text { Piper aduncum L. }\end{array}$ & 1087,359 & 147,832 \\
\hline Trolox $^{\circledR}$ a & 2,0 & 2,68 \\
\hline
\end{tabular}

${ }^{a}$ Estándar de referencia

Tabla 3. Actividad antioxidante del aceite esencial de Piper aduncum L. expresada como capacidad antioxidante equivalente a Trolox (TEAC).

\begin{tabular}{lccc}
\hline & \multicolumn{3}{c}{ TEAC } \\
\cline { 2 - 4 } Ensayo & $\boldsymbol{\mu g}$ Trolox/mg & $\mathbf{m g}$ Trolox/mL & $\boldsymbol{\mu m o l ~ T r o l o x} / \mathbf{m L}$ \\
& 1,839 & 1,824 & 7,286 \\
DPPH $^{\bullet}$ & 17,79 & 17,64 & 70,509 \\
ABTS $^{\bullet+}$ & & & \\
\hline
\end{tabular}

Las figuras 2 y 3 muestran el comportamiento del aceite esencial de Piper aduncum L. como captador de radicales DPPH y ABTS, respectivamente. Las concentraciones del aceite esencial se ajustaron linealmente con los valores de \% inhibición y los coeficientes de determinación $\left(\mathrm{R}^{2}\right)$ fueron mayores a 0,9 . Estos valores demuestran que la concentración de aceite esencial y el porcentaje de inhibición presentan una correlación lineal significativa. 


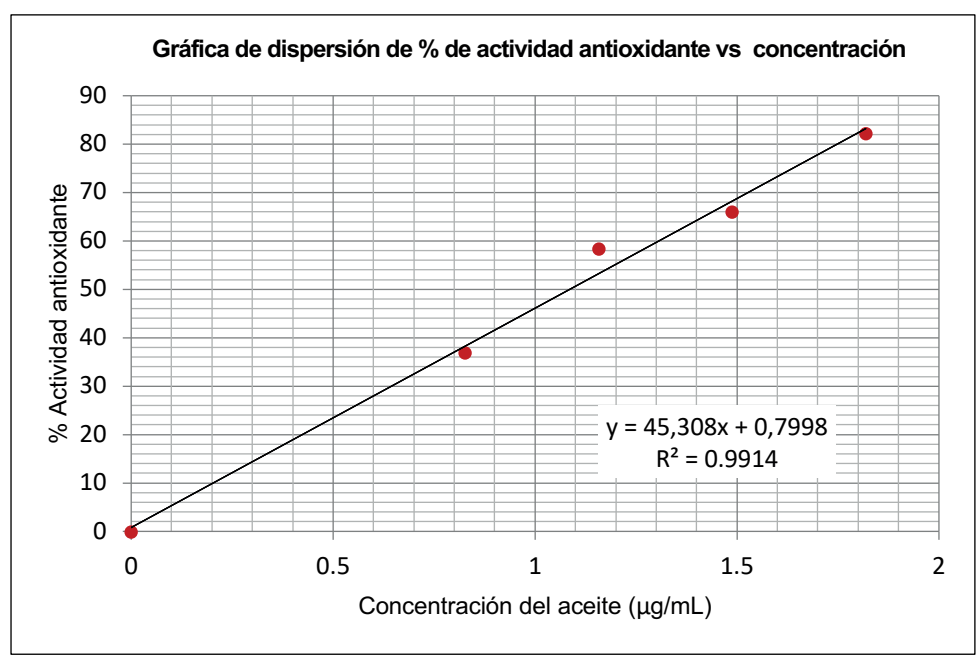

Figura 2. Curva de captación del radical DPPH del aceite esencial de Piper aduncum L.

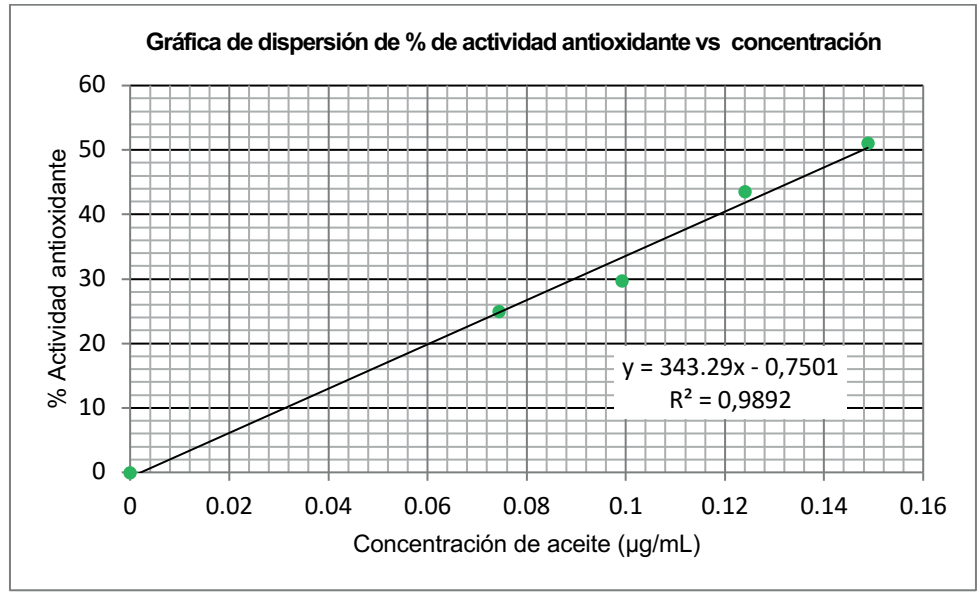

Figura 3. Curva de captación del radical ABTS del aceite esencial de Piper aduncum L. 


\section{Efecto fungistático del aceite esencial}

El resultado muestra que la susceptibilidad de C. albicans frente al aceite esencial de Piper aduncum L. fue baja en comparación con el ketoconazol (tabla 4). La diferencia puede deberse a los diferentes mecanismos relacionados con sus estructuras. Esta facultad en aceite esencial de Piper aduncum L. se atribuiría a los componentes minoritarios, como germacreno $\mathrm{D}$, biciclogermacreno, $\beta$-cariofileno, $\beta$-ocimeno y alcanfor ${ }^{16,17}$. Su efecto fungistático puede explicarse por las propiedades lipofílicas de estos metabolitos, que les permite interrumpir la membrana celular de ergosterol o destruir la mitocondria fúngica ${ }^{17}$.

Tabla 3. Efecto fungistático del aceite esencial de Piper aduncum L., expresado como concentración mínima inhibitoria (MIC).

\begin{tabular}{cc}
\hline \multirow{2}{*}{ Muestra } & $\begin{array}{c}\text { Actividad inhibitoria frente a } \\
\text { cepa fúngica } \\
\text { Candida albicans }\end{array}$ \\
\cline { 2 - 2 } & MIC $(\mathbf{m g} / \mathbf{m L})$ \\
\hline Piper aduncum $\mathrm{L}$. & 1,24 \\
Ketoconazol $^{\mathrm{a}}$ & $0,08^{\mathrm{b}}$ \\
\hline
\end{tabular}

${ }^{a}$ Estándar de referencia

${ }^{\mathrm{b}}$ Unidad en $\mu \mathrm{g} / \mathrm{mL}$

Por otro lado, no hay suficientes estudios sobre las actividades antioxidante y fungistática de 1,2,4-trimetoxi-5-(1-propenil)-benceno y metileugenol. Las investigaciones de estos compuestos están principalmente dirigidos a evaluar su actividad antiparasitaria, antiprotozoaria y antitumoral ${ }^{4}$.

\section{CONCLUSIONES}

Un total de 35 componentes fueron identificados en el aceite esencial de Piper aduncum L. "matico", de los cuales ocho representan el 76,53\% del contenido total. Los principales componentes fueron: 1,2,4-trimetoxi-5-(1-propenil)-benceno, metileugenol, germacreno $\mathrm{D}$, biciclogermacreno, 4,7,7-trimetilbiciclo [2.2.1] heptan-3-ona, $\beta$-cariofileno, $\delta$ - cadineno y $\beta$-ocimeno. El aceite esencial de Piper aduncum L. "matico" mostró limitada actividad antioxidante como captador de radicales DPPH y ABTS comparado con el antioxidante de referencia. En cuanto al efecto fungistático el aceite esencial de Piper aduncum L. "matico" también fue limitado frente a Candida albicans ATCC 10231. Sin embargo, el alto contenido de fenilpropanoides lo hace un candidato prometedor para futuras investigaciones. 


\section{AGRADECIMIENTOS}

Al Vicerrectorado de Investigación y Posgrado por el financiamiento para la realización del trabajo, al Instituto de Investigación en Ciencias Farmacéuticas y Recursos Naturales "Juan de Dios Guevara” y al Instituto de Química Biológica, Microbiología y Biotecnología "Marco Antonio Garrido Malo" de la Facultad de Farmacia y Bioquímica de la Universidad Nacional Mayor de San Marcos; en la culminación del presente estudio,

\section{REFERENCIAS BIBLIOGRÁFICAS}

1. Brewer M. Natural Antioxidants: Sources, compounds, mechanisms of action, and potential applications. Compr Rev Food Sci Food Saf. 2011; 10(4):221-247.

2. Prasad R. Candida albicans: Cellular and Molecular Biology. 2nd ed. Cham, Switzerland: Springer International Publishing AG; 2017.

3. Laxminarayan R, Matsoso P, Pant S, Brower C, Røttingen JA, Klugman K, et al. Access to effective antimicrobials: a worldwide challenge, Lancet. 2016; 387(10014):168-75.

4. Di Liberto M, Svetaz L, Castelli M, Rai M, Derita M. Role of Essential Oils for the Cure of Human Pathogenic Fungal Infections. In: Rai M, Zacchino S, Derita M, editors. Essential Oils and Nanotechnology for Treatment of Microbial Diseases. Boca Raton, Florida: CRC Press, Taylor \& Francis Group; 2018. p.128-38.

5. León B. Piperaceae endémicas del Perú. Rev Perú Biol. 2006; 13(2):492-563.

6. Kloucek P, Polesny Z, Svobodova B, Vlkova E, Kokoska L. Antibacterial screening of some Peruvian medicinal plants used in Callería District. J. Ethnopharmacol. 2005; 99(2): 309-12.

7. Valadeau C, Pabon A, Deharo E, Albán-Castillo J, Yannick E, Lores FA, et al. Medicinal plants from the Yanesha (Perú): Evaluation of the leishmanicidal and antimalarial activity of selected extracts. J Ethnopharmacol. 2009; 123(3): 413-22.

8. Brand-Williams W, Cuvelier ME, Berset C. Use of a free-radical method to evaluate antioxidant activity. LWT Food Sci Technol. 1995; 28(1): 25-30.

9. Re R, Pellegrini N, Proteggente A, Pannala A. Antioxidant activity applying an improved ABTS radical cation decolorization assay. Free Radic Biol Med. 1999; 26(9-10):1231-7.

10. Clinical Laboratory Standards Institute. Reference method for broth dilution antifungal susceptibility testing of yeasts; Approved Standard - 3rd ed. CLSI document M27-A3. Wayne, PA: Clinical and Laboratory Standards Institute; 2008.

11. Liu M, Seidel V, Katerere DR, Gray AI. Colorimetric broth microdilution method for the antifungal screening of plant extracts against yeasts. Methods. 2007; 42(4):325-9.

12. Durant-Archibold A, Santana A, Gupta M, Ethnomedical uses and pharmacological activities of most prevalent species of genus Piper in Panama:AReview. JEthnopharmacol. 2018; 10(217): 63-82.

13. Schmidt E. Production of Essential Oils. In: Baser CKH, Buchbauer G, editors. Handbook of essential oils: science, technology, and applications. Boca Raton: CRC Press, Taylor \& Francis Group; 2010. p. 83-118. 
14. Zaluski D, Cielsa L, Janeczko Z. The Structure-Activity Relationships of Plant Secondary Metabolites with Antimicrobial, Free Radical Scavenging and Inhibitory Activity toward Selected Enzymes. In: Atta-ur-Rahman FRS, editor. Studies in Natural Products Chemistry. 2015; 45:217-49. doi: 10.1016/B978-0-444-63473-3.00007-1.

15. Bakkali F, Averbeck S, Averbeck D, Idaomar M. Biological effects of essential oils - A review. Food Chem Toxicol. 2008; 46(2): 446-75.

16. Da Silva J, Pinto L, Burbano R, Montenegro R, Guimarães E, Andrade E, et al. Essential oils of Amazon Piper species and their cytotoxic, antifungal, antioxidant and anticholinesterase activities. Industrial Crops and Products. 2014; 58: 55-60.

17. Nazzaro F, Fratianni F, Coppola R, De Feo V. Essential Oils and Antifungal Activity. Pharmaceuticals 2017; 10(4): 86. 